\title{
MSI2 knockdown represses extrahepatic cholangiocarcinoma growth and invasion by inhibiting epithelial-mesenchymal transition
}

This article was published in the following Dove Press journal:

OncoTargets and Therapy

\author{
Feihu $\mathrm{Hu}^{1,2}$ \\ Chenhai Liu' ${ }^{1,2}$ \\ Fang $X_{i e^{1,2}}$ \\ Xiansheng $\operatorname{Lin}^{1,2}$ \\ Ji Yang ${ }^{1,2}$ \\ Chao Wang ${ }^{1,2}$ \\ Qiang Huang ${ }^{1,2}$ \\ 'Department of General Surgery, \\ Anhui Provincial Hospital Affiliated to \\ Anhui Medical University, Hefei, China; \\ ${ }^{2}$ Anhui Province Key Laboratory of \\ Hepatopancreatobiliary Surgery, Anhui \\ Provincial Hospital, Heifei, China
}

Purpose: To investigate the expression and functional role of Musashi2 (MSI2), an RNA-binding protein, in extrahepatic cholangiocarcinoma (eCCA).

Patients and methods: We measured MSI2 expression in human specimens and cell lines using Western blot and quantitative real-time polymerase chain reaction, and we analyzed its association with clinicopathologic features in eCCA patients. Univariate and multivariate analyses were performed to identify risk factors correlated with overall survival and diseasefree survival. Functional experiments were used to study the mechanisms of MSI2 in regulating eCCA cell growth, migration, and invasion.

Results: MSI2 expression was upregulated significantly in both human specimens and cell lines, and high MSI2 expression was associated with lymph node metastasis, advanced TNM stage, and poor prognosis in eCCA patients. Additionally, MSI2 overexpression promoted eCCA cell growth, migration, and invasion, while MSI2 knockdown repressed eCCA cell migration and invasion by inhibiting epithelial-mesenchymal transition.

Conclusion: MSI2 is an independent prognostic factor for eCCA patients, and MSI2 downregulation inhibits eCCA cell growth and metastasis. MSI2 may be a potential therapeutic target for eCCA patients.

Keywords: Musashi2, cholangiocarcinoma, prognosis, metastasis, EMT

\section{Introduction}

Cholangiocarcinoma (CCA) is an epithelial cell malignancy originating from various locations of the biliary tree. According to its anatomical location, it can be classified into intrahepatic and extrahepatic subtypes. ${ }^{1}$ Extrahepatic cholangiocarcinoma (eCCA) accounts for approximately $90 \%$ of cholangiocarcinoma cases; most of these are moderately poorly differentiated adenocarcinomas, and other histological subtypes are encountered rarely. ${ }^{2-4}$ The highly desmoplastic nature of eCCA and its extensive support by rich tumor microenvironments contribute to its therapeutic resistance. Surgical resection is the only preferred treatment for eCCA. Unfortunately, most patients are not suitable for curative resection since the disease is generally too advanced at the time of diagnosis. ${ }^{5,6}$ Although liver transplantation and surgical resection are choices for patients with eCCA, the 5-year survival rate is very low..$^{7-9}$ Therefore, identifying effective prognostic biomarkers and therapeutic approaches is crucial for improving the diagnosis and survival of eCCA.

The Musashi (MSI) gene belongs to the RNA-binding protein family, which participates in posttranscriptional regulation by binding to target mRNAs. ${ }^{10,11}$
Correspondence: Qiang Huang Department of General Surgery, Affiliated Provincial Hospital, Anhui Medical University, 17 Lujiang Road, Luyang, Hefei, Anhui 23000I, China

Tel +8655I 62283320

Email198538838@qq.com 
In mammals, the evolutionarily conserved MSI gene has two homologs: MSI1 and MSI2. ${ }^{12}$ In recent years, MSI2 was reported to play an important role in various malignant tumors. For example, MSI2 acts as an essential regulator of hematopoietic stem cell self-renewal, and it was reported to be a potent cooperative oncogene in human leukemia. ${ }^{13,14}$ High MSI2 expression can induce a more aggressive myeloid form of the disease, whereas MSI2 abrogation can promote differentiation, decrease proliferation, and increase apoptosis in myeloid leukemia cells. ${ }^{13,15,16}$ Researchers have also demonstrated the roles of MSI2 in solid tumor progression and development. He et al reported that MSI2 promoted the invasion and progression of hepatocellular carcinoma by interacting with the Wnt/beta-catenin pathway and inducing epithelial-mesenchymal transition (EMT). ${ }^{17}$ Another study found that MSI2 contributed to the invasion of lung adenocarcinoma through TGF- $\beta$ signaling and that it may serve as a predictive biomarker of non-small-cell lung cancer aggressiveness. ${ }^{18}$ Moreover, MSI2 overexpression has been linked to therapeutic resistance in cancer, and some groups are attempting to develop small-molecule inhibitors of this protein. ${ }^{19-21}$ However, to the best of our knowledge, the expression and functional role of MSI2 in eCCA have not been investigated.

In the present study, we sought to evaluate MSI2 expression in human specimens, determine the association between MSI2 expression and clinicopathological characteristics, and identify the functional role of MSI2 in the growth and metastasis of eCCA cells.

\section{Patients and methods Patients and follow-up}

Seventy-eight paraffin-embedded specimens pathologically diagnosed as eCCA and matched adjacent normal bile duct tissues were obtained from the specimen bank of the pathology department of Anhui Provincial Hospital. All patient clinicopathological data, including sex, age, tumor size, histological grade, and lymph node metastasis, were available. Both overall survival (OS) and disease-free survival (DFS) were acquired to evaluate the prognostic value of MSI2 in patients after surgery. The median follow-up period was 19.5 months, ranging from 1 to 60 months. This study was performed in accordance with the Helsinki Declaration and was approved by the Human Research Ethics Committee of Anhui Provincial Hospital. Written informed consent was obtained from each patient for this study.

\section{Immunohistochemistry}

Immunohistochemical staining of paraffin-embedded specimens was performed with primary antibodies against MSI2 (ab76148, Abcam), as previously described. ${ }^{22}$ The staining intensity was classified as negative ( 0 points), weak ( 1 point), moderate ( 2 points), or strong ( 3 points). The percentage of stained cells was defined as negative ( 0 points), $<10 \%$ ( 1 point), $10 \%-50 \%$ ( 2 points), or $>50 \%$ (3 points). The final score was the sum of the extent and intensity of staining. A total score $\leq 3$ was regarded as low expression; otherwise, the scores were considered to indicate high expression.

\section{Cell lines}

A human normal biliary epithelial cell line (HIBEpiC) and eCCA cell lines (QBC939 and RBE) were purchased from the Cell Bank of the Chinese Academy of Sciences (Shanghai, China). All cell lines were cultured in RPMI-1640 medium containing $10 \%$ fetal bovine serum and $1 \%$ penicillin/ streptomycin.

\section{Quantitative real-time PCR}

Total RNA was extracted from tissues and cell lines to synthesize cDNA according to the manufacturer's protocol. Then, quantitative real-time polymerase chain reaction (qRT-PCR) was conducted using SYBR Green reagent and an ABI Prism 7900HT sequence detection system (Applied Biosystems, Thermo Fisher Scientific). The primers used in our study were designed as follows: MSI2, (forward) 5'-ACCTCACCAGATAGCCTTAGAG-3' and (reverse) 5'-AGCGTTTCGTAGTGGGATCTC-3'; GAPDH, (forward) 5'-CCTAGTTCGTCATGGGTGTGAACCA-3' and (reverse) 5'-GCCAGTAGAGGCAGGGATGATGTTC-3'.

\section{Western blot assay}

Tissues and cells were lysed in cell lysis buffer supplemented with protease inhibitor cocktail on ice for 30 minutes. Then, the samples were centrifuged at 12,000 rpm for 15 minutes, and the supernatants containing the proteins were collected. The proteins were separated by SDS-PAGE and transferred onto polyvinylidene difluoride membranes. Then, the membranes were blocked with 5\% skim milk for 1 hour at room temperature and incubated with the primary antibodies at $4^{\circ} \mathrm{C}$ overnight. The next day, after the membranes were incubated with horseradish peroxidase-conjugated secondary antibody for 1 hour, the bands were captured and visualized with a chemiluminescence imaging system (Shanghai, China). 
The primary antibodies used were as follows: anti-MSI2 (ab76148, Abcam); anti-E-cadherin (ab1416, Abcam); antivimentin (ab8978, Abcam); and anti-N-cadherin (ab76011, Abcam).

\section{Plasmids and lentiviral infection}

The MSI2-shRNA plasmid was a gift from the University of Science and Technology of China. Briefly, the MSI2-shRNA vectors were cotransfected with lentiviral packing vectors, pMD2.G and psPAX2, into 293 T cells. Forty-eight hours later, the virus particles were harvested, filtered, and used to infect eCCA cells. Polybrene was also added to a final concentration of $8 \mu \mathrm{g} / \mathrm{mL}$. Finally, puromycin $(2 \mu \mathrm{g} / \mathrm{mL})$ was used for $>2$ weeks to select the resistant cells.

\section{Colony formation assay}

Cells were plated in six-well plates $(1,000$ per well) and allowed to grow in culture medium. After 2 weeks, the colonies were fixed in $4 \%$ paraformaldehyde for 20 minutes, and then stained with $0.1 \%$ crystal violet solution for 50 minutes. Colonies with more than 20 cells were counted using an inverted microscope.

\section{Cell Counting Kit-8 assay}

Cell Counting Kit-8 (CCK-8) assays were used to evaluate the proliferative ability of eCCA cells. Briefly, cells were seeded into 96 -well plates $\left(5 \times 10^{4}\right.$ cells per well). After 0,24 , 48,72 , and 96 hours of incubation, $10 \mu \mathrm{L}$ of CCK-8 solution was added. Two hours later, the $\mathrm{OD}_{450}$ was measured in triplicate using a microplate reader. Cell growth curves were drawn to assess the proliferative ability of the cells.

\section{Cell migration and invasion assays}

For the wound healing assays, cells were seeded in six-well plates and allowed to grow to confluence. A $200-\mu \mathrm{L}$ sterile tip was used to create a wound in each well. Then, the cells were cultured in serum-free medium for 48 hours. The cells that migrated into the wound area were observed and photographed with an inverted microscope. For the Boyden chamber assays, 24-well chambers with 12 cell culture inserts, which contained $8 \mu \mathrm{m}$ pore polycarbonate membranes precoated with (for the invasion assay) or without (for the migration assay) Matrigel, were used. A total of $200 \mu \mathrm{L}$ of serum-free medium containing $5 \times 10^{4}$ cells was added to the upper chamber, and $500 \mu \mathrm{L}$ of culture medium containing $10 \%$ fetal bovine serum was added to the lower chamber. After 48 hours of incubation, the cells attached to the lower surface of the membrane were fixed in methanol for 20 minutes and stained with a $0.1 \%$ crystal violet solution.

\section{Statistical analysis}

SPSS 22.0 (IBM Corporation, Armonk, NY, USA) and GraphPad Prism 7 (San Diego, CA, USA) software were used for statistical analyses. Student's $t$-test was used to estimate significant differences between groups. The correlations between MSI2 expression and the clinicopathologic characteristics were assessed by the two-tailed chi-squared test or Fisher's exact test. The associations between the MSI2 expression level and patient survival were analyzed with the Kaplan-Meier method and compared using the log-rank test. A multivariate Cox regression model was used to find independent factors of prognosis. $P \leq 0.05$ was considered to be statistically significant.

\section{Results}

\section{MSI2 expression is upregulated in eCCA tissues}

We evaluated MSI2 mRNA expression in 20 pairs of human specimens using qRT-PCR. As shown in Figure 1A, MSI2 mRNA expression was significantly higher in tumor tissues than in adjacent normal tissues $(4.00 \pm 0.26$ vs $2.80 \pm 0.24$, $P=0.0015)$. MSI2 protein expression levels were detected using Western blot assays. Consistent with the qRT-PCR data, MSI2 protein levels were also significantly higher in eCCA tissues than in normal tissues $(1.68 \pm 0.12$ vs $1.11 \pm 0.07$, $P=0.0002$; Figure $1 \mathrm{~B}$ and $\mathrm{C}$ ). In addition, we performed immunohistochemical staining on 78 eCCA samples, and the results further confirmed the high MSI2 expression levels in tumor tissues (Figure 1D and Table S1).

\section{MSI2 upregulation predicts poor prognosis in eCCA}

To investigate the relationship between the MSI2 expression and the clinicopathological features of eCCA patients, 78 eCCA patients were classified into low and high MSI2 expression subgroups according to the immunohistochemical score. As shown in Table 1, high MSI2 expression was positively correlated with lymph node metastasis $(P=0.028)$ and advanced TNM stage $(P=0.008)$. In addition, KaplanMeier analyses were performed to evaluate the prognostic value of MSI2. The results showed that high MSI2 expres-

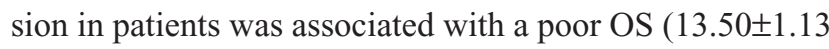
vs $42.00 \pm 7.47$ months, $P<0.001)$ and DFS $(11.30 \pm 1.15$ vs 31.20 \pm 6.81 months, $P<0.001$; Figure 2). Moreover, 


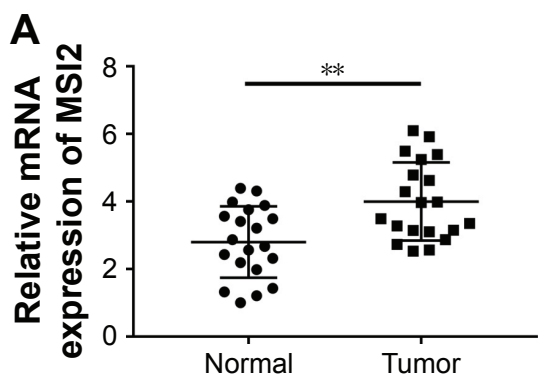

D

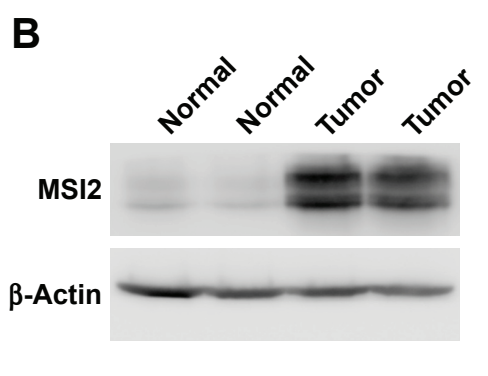

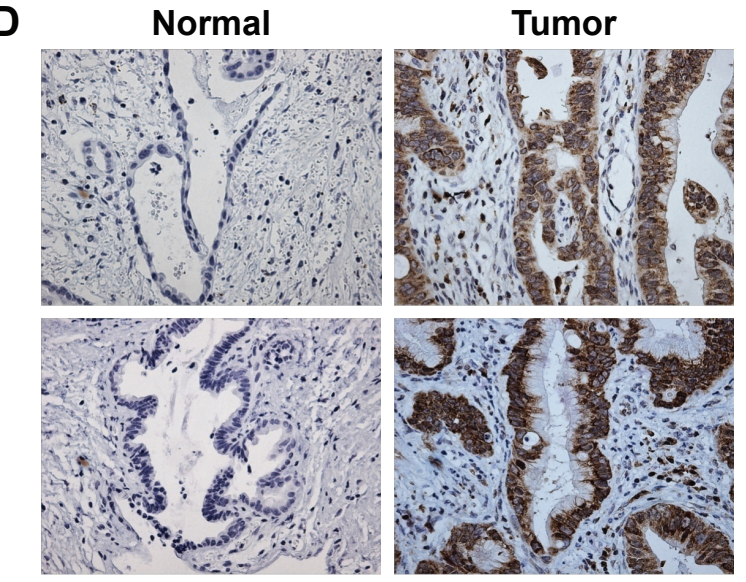
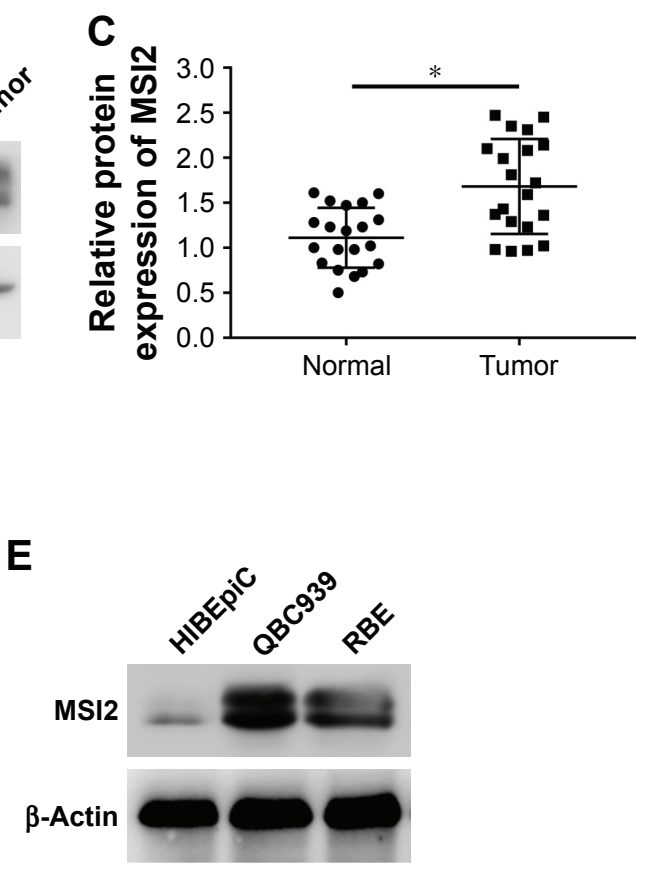

Figure I MSI2 is overexpressed in extrahepatic cholangiocarcinoma.

Notes: (A) qRT-PCR and (C) Western blot analysis of MSI2 expression in 20 pairs of eCCA tissues and matched adjacent normal tissues. (B) Representative images of MSI2 protein expression in eCCA tissues and paired normal tissues from two patients. (D) Representative images of IHC staining for MSI2 with paraffin-embedded sections. Magnification 200×. (E) MSI2 expression in human normal biliary epithelial cells (HIBEpiC) and eCCA cell lines (QBC939 and RBE). $* P<0.05$, and $* * P<0.01$.

Abbreviations: eCCA, extrahepatic cholangiocarcinoma; IHC, immunohistochemistry; qRT-PCR, quantitative real-time polymerase chain reaction.

multivariate analyses indicated that high MSI2 expression was an independent prognostic factor for $\mathrm{OS}(\mathrm{HR}=1.805$, 95\% CI: $1.050-3.105, P=0.033)$ and DFS (HR=1.845, 95\% CI: $1.072-3.178, P=0.027$ ) in eCCA patients (Table 2).

\section{MSI2 knockdown inhibited eCCA cell proliferation and growth}

To investigate the effect of MSI2 on eCCA cell growth and proliferation, we first analyzed its expression in eCCA cell lines. The results revealed that MSI2 expression was dramatically higher in the eCCA cell lines QBC939 and RBE than in the normal bile duct epithelial cell HIBEpiC (Figure 1E). Therefore, we knocked down MSI2 in the QBC939 cell line (Figure 3A) and then performed colony formation assays. As shown in Figure 3B and C, MSI2 downregulation inhibited eCCA cell growth and colony formation ability. In addition, CCK-8 assays were performed, which also indicated that MSI2 downregulation repressed eCCA cell growth and proliferation (Figure 3D). All these results were further confirmed in RBE cell line (Figure S1). Taken together, these data suggest that MSI2 may regulate the translation of proteins that controlled eCCA cell growth.

\section{MSI2 promotes eCCA cell migration and invasion}

To determine the effect of MSI2 on eCCA cell migration and invasion, we first performed wound healing assays using QBC939 cell line. Compared with the control group, MSI2 knockdown reduced the ability of eCCA cell to migrate to the scratched area (Figure 4A and B). Next, we performed Boyden chamber assays. Consistent with the above results, MSI2 downregulation decreased the migratory ability of eCCA cells. In addition, the chamber assays also demonstrated that MSI2 downregulation markedly decreased eCCA cell invasiveness (Figure 4C and D). These data were further confirmed in RBE cell line (Figure S2).

\section{MSI2 downregulation inhibits epithelial- mesenchymal transition in eCCA}

EMT, a reversible dynamic process through which epithelial cells gain the characteristics of mesenchymal cells, is a wellknown early event of metastasis in malignant tumors. ${ }^{23-25}$ The main features of the EMT process are decreased expression of the epithelial marker E-cadherin and increased expression of the mesenchymal markers $\mathrm{N}$-cadherin and vimentin. ${ }^{26}$ 
Table I Correlation between MSI2 expression and clinicopathologic characteristics of extrahepatic cholangiocarcinoma

\begin{tabular}{|c|c|c|c|c|}
\hline \multirow[t]{2}{*}{ Parameter } & \multirow[t]{2}{*}{$\mathbf{n}$} & \multicolumn{2}{|c|}{$\begin{array}{l}\text { MSI2 } \\
\text { expression }\end{array}$} & \multirow[t]{2}{*}{$P$-value } \\
\hline & & Low & High & \\
\hline Age (years) & & & & 0.367 \\
\hline$\leq 60$ & 27 & 14 & 13 & \\
\hline$>60$ & 51 & 21 & 30 & \\
\hline Gender & & & & 0.747 \\
\hline Male & 43 & 20 & 23 & \\
\hline Female & 35 & 15 & 20 & \\
\hline Tumor size & & & & 0.828 \\
\hline$\leq 3 \mathrm{~cm}$ & 57 & 26 & 31 & \\
\hline$>3 \mathrm{~cm}$ & 21 & 9 & 12 & \\
\hline Tumor location & & & & 0.789 \\
\hline Perihilar & 50 & 23 & 27 & \\
\hline Distal & 28 & 12 & 16 & \\
\hline Differentiation & & & & 0.506 \\
\hline Well + moderate & 62 & 29 & 33 & \\
\hline Poor & 16 & 6 & 10 & \\
\hline Lymph node metastasis & & & & 0.028 \\
\hline Absent & 60 & 31 & 29 & \\
\hline Present & 18 & 4 & 14 & \\
\hline Perineural invasion & & & & 0.100 \\
\hline Absent & 41 & 22 & 19 & \\
\hline Present & 37 & 13 & 24 & \\
\hline TNM stage & & & & 0.008 \\
\hline I-II & 55 & 30 & 25 & \\
\hline III-IV & 23 & 5 & 18 & \\
\hline
\end{tabular}

Abbreviation: TNM, tumor-node-metastasis.
To investigate whether MSI2 promoted eCCA migration and invasion by inducing EMT, we first evaluated E-cadherin expression in human specimens using immunohistochemistry. Consistent with our prediction, E-cadherin expression was markedly downregulated in eCCA tissues compared with normal bile duct tissue (Figure 5A). Spearman rank correlation test showed that MSI2 expression was negatively associated with E-cadherin expression $(r=-0.586, P<0.001$; Table 3). We then assessed EMT-related markers expression in eCCA cell lines using Western blot assays. As shown in Figure 5B and C, MSI2 knockdown increased E-cadherin expression and decreased $\mathrm{N}$-cadherin and vimentin expressions. Collectively, our results demonstrated that MSI2 could promote eCCA migration and invasion through, at least partially, inducing EMT.

\section{Discussion}

The MSI2 and MSI1 RNA-binding proteins are classified as subgroup of the class A/B heterogeneous nuclear ribonucleoproteins. ${ }^{10}$ In the N-terminal of the MSI2 protein, there are two highly conserved RNA recognition motifs: RRM1 and RRM2. These motifs bind to a series of target mRNAs to regulate posttranscriptional translation. In addition, in the C-terminus of this protein, there are auxiliary sequences that mediate protein-protein interactions and allow the protein
A

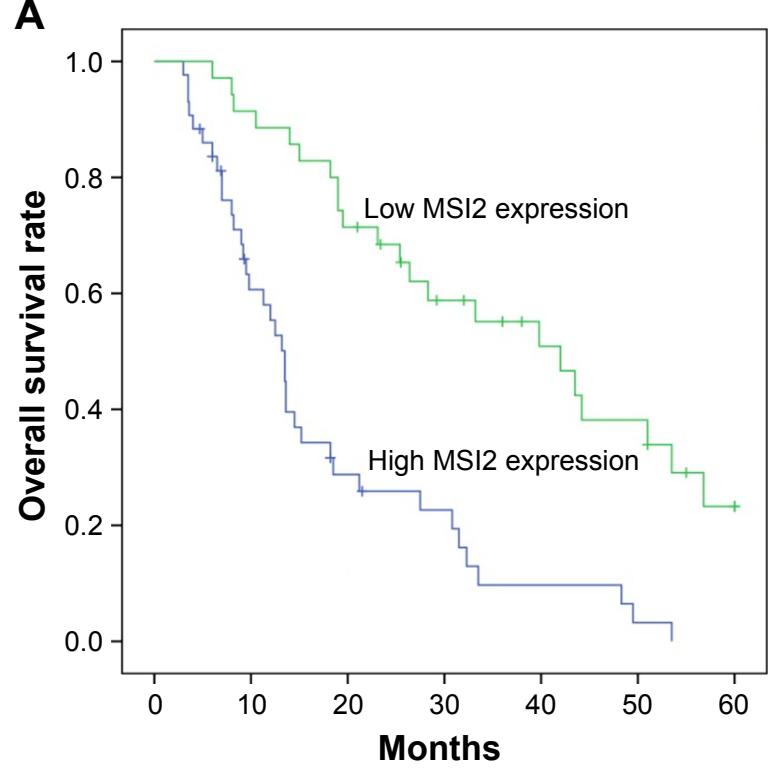

B

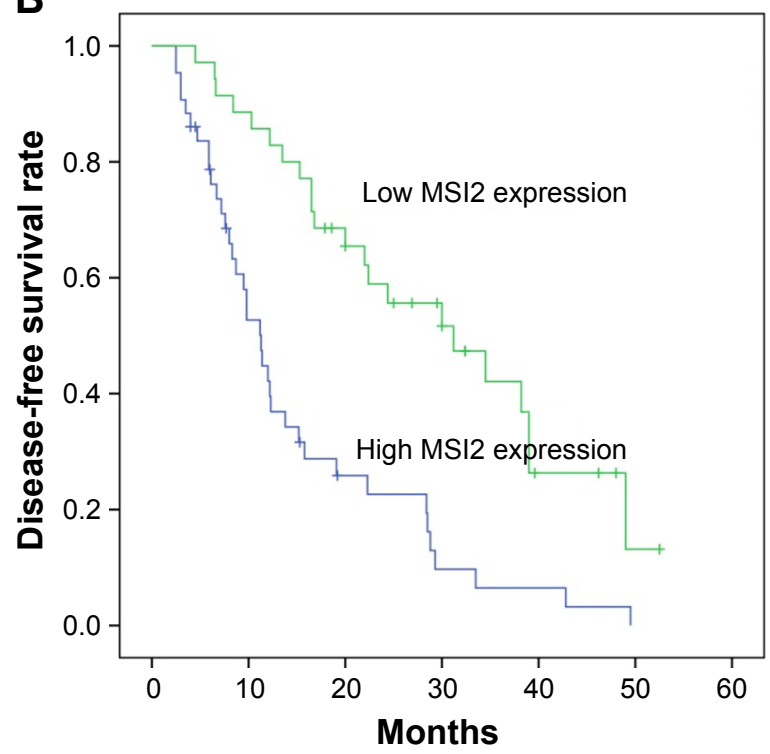

$$
\neg \text { High MSI2 expression } \neg \text { Low MSI2 expression }+ \text { Censored }+ \text { Censored }
$$

Figure 2 Kaplan-Meier survival analysis of association between MSI2 expression and prognosis of patients with extrahepatic cholangiocarcinoma.

Notes: (A) Patients with high MSI2 expression has a shorter OS compared with patients with low MSI2 expression ( $n=78, P<0.001)$. (B) Patients with high MSI2 expression has shorter DFS compared with patients with low MSI2 expression $(n=78, P<0.001)$.

Abbreviations: DFS, disease-free survival; OS, overall survival. 
Table 2 Univariate and multivariate analysis of factors associated with OS and DFS

\begin{tabular}{|c|c|c|c|c|c|c|}
\hline \multirow[t]{2}{*}{ Parameter } & \multicolumn{3}{|c|}{ Overall survival } & \multicolumn{3}{|c|}{ Disease-free survival } \\
\hline & HR & $95 \% \mathrm{Cl}$ & $P$-value & HR & $95 \% \mathrm{CI}$ & $P$-value \\
\hline \multicolumn{7}{|l|}{ Univariate analysis } \\
\hline Age ( $\leq 60$ vs $>60$ years $)$ & 0.914 & $0.551-1.518$ & 0.729 & 0.906 & $0.547-1.502$ & 0.703 \\
\hline Gender (male vs female) & 0.932 & $0.572-1.52 \mid$ & 0.779 & 0.935 & $0.573-1.528$ & 0.789 \\
\hline Tumor size $(\leq 3$ vs $>3 \mathrm{~cm})$ & 0.949 & $0.530-1.697$ & 0.859 & 0.969 & $0.54 I-1.737$ & 0.917 \\
\hline Tumor location (perihilar vs distal) & 0.795 & $0.484-1.306$ & 0.365 & 0.808 & $0.491-1.332$ & 0.403 \\
\hline Perineural invasion (yes vs no) & 1.826 & $1.115-2.989$ & 0.018 & $\mathrm{I} .802$ & $1.10 \mathrm{I}-2.950$ & 0.019 \\
\hline Differentiation (well/moderate vs poor) & 0.827 & $0.501-1.366$ & 0.458 & 0.806 & $0.489-1.327$ & 0.396 \\
\hline TNM stage (III/IV vs I/II) & 5.130 & $2.805-9.381$ & $<0.001$ & 5.238 & $2.852-9.622$ & $<0.001$ \\
\hline Lymph node metastasis (yes vs no) & 2.064 & $1.152-3.696$ & 0.015 & 2.067 & I.153-3.705 & 0.015 \\
\hline MSI2 expression (high vs low) & 2.462 & I.487-4.074 & $<0.001$ & 2.476 & $1.494-4.10 \mathrm{I}$ & $<0.001$ \\
\hline \multicolumn{7}{|l|}{ Multivariate analysis } \\
\hline Perineural invasion (yes vs no) & 1.460 & $0.832-2.561$ & 0.187 & 1.416 & $0.808-2.484$ & 0.225 \\
\hline TNM stage (III/IV vs I/II) & 4.464 & $2.362-8.438$ & $<0.001$ & 4.548 & $2.395-8.637$ & $<0.001$ \\
\hline Lymph node metastasis (yes vs no) & 1.064 & $0.549-2.063$ & 0.853 & 1.061 & $0.546-2.062$ & 0.861 \\
\hline MSI2 expression (high vs low) & 1.805 & $1.050-3.105$ & 0.033 & $\mathrm{I} .845$ & $1.072-3.178$ & 0.027 \\
\hline
\end{tabular}

Abbreviations: DFS, disease-free survival; HR, hazard ratio; OS, overall survival; TNM, tumor-node-metastasis.

to both induce and suppress translation. ${ }^{27-29}$ In recent years, the understanding of the biochemical function of the MSI2 protein structure has increased, and numerous studies have reported the essential role of MSI2 in regulating various critical biological processes relevant to cancer initiation and progression. For example, Bennett et al identified a number of MSI2 targets by using transcriptome-wide binding assays. Of these identified targets, most were key regulators governing
A

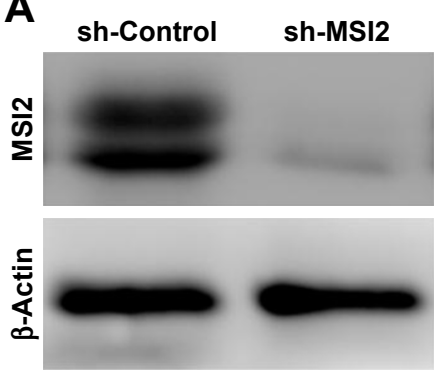

C

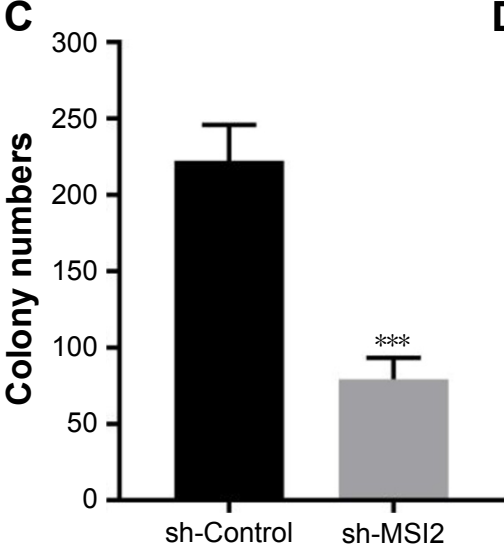

B

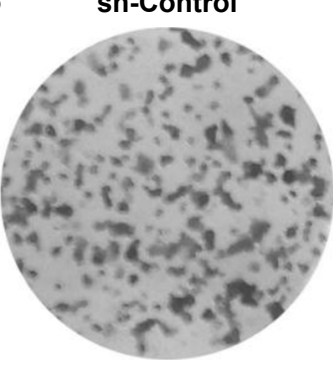

D

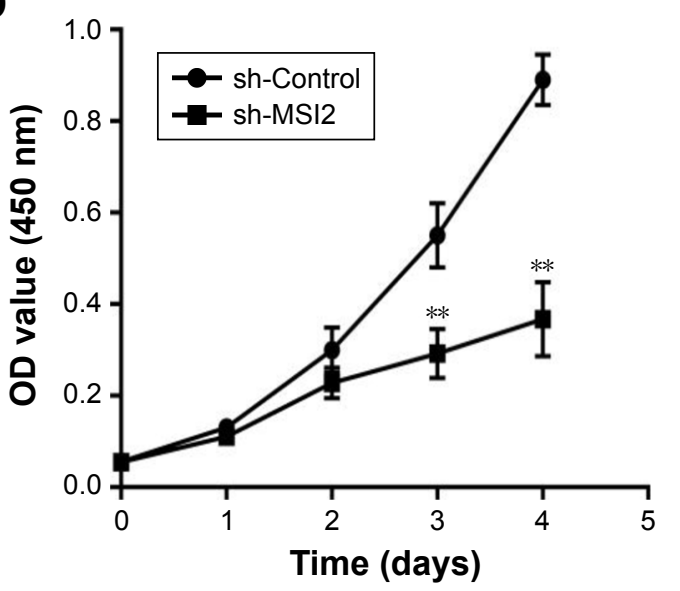

Figure 3 MSI2 knockdown inhibits extrahepatic cholangiocarcinoma cell growth.

Notes: (A) Verification of the efficiency of MSI2-shRNA plasmid in QBC939 cell line. (B) Colony formation assays show the effect of MSI2 on QBC939 cell growth and colony formation. (C) Quantification of colony formation assays through calculating colony numbers. (D) CCK-8 assay shows the effect of MSI2 on QBC 939 cell proliferation. $* * P<0.01$, and $* * * P<0.001$.

Abbreviations: CCK-8, Cell Counting Kit-8; OD, optical density. 
A
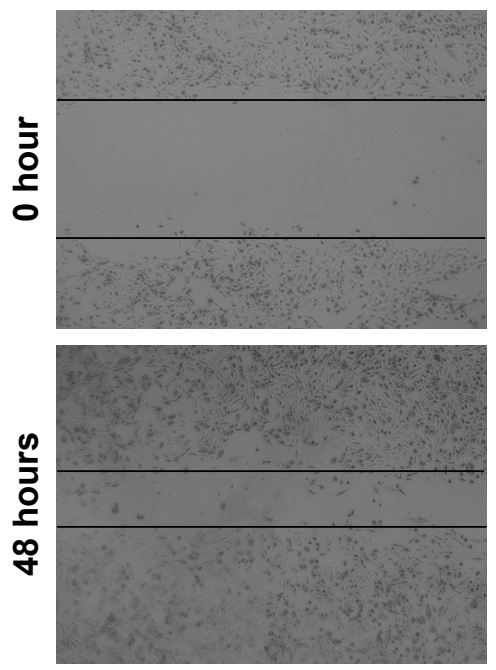

C

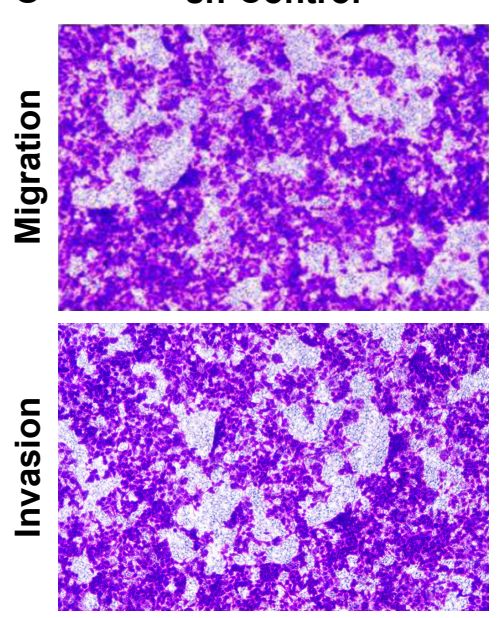

sh-MSI2
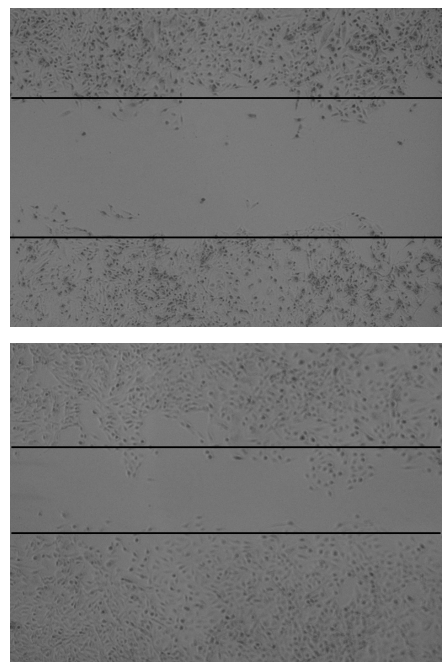

sh-MSI2

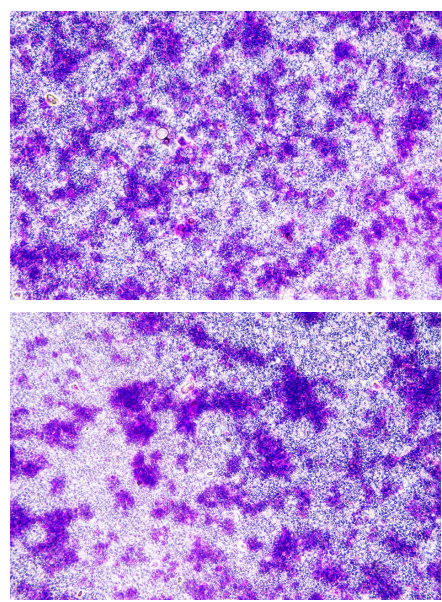

B

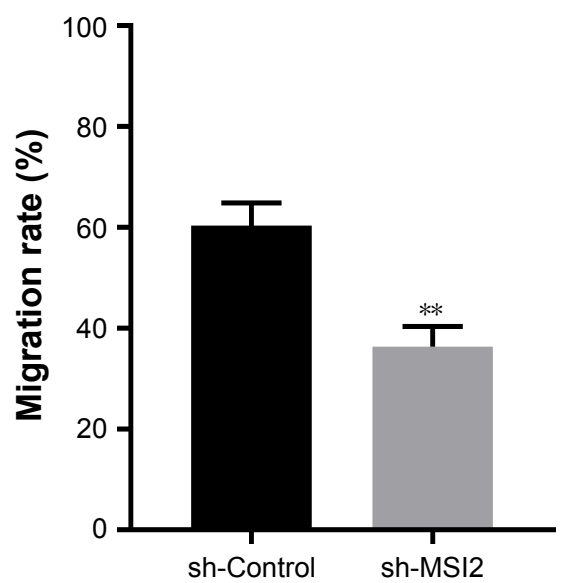

D

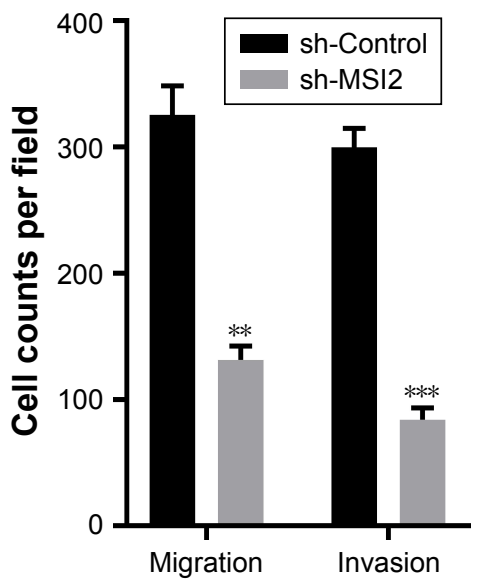

Figure 4 MSI2 promotes the migration and invasion of extrahepatic cholangiocarcinoma.

Notes: (A) Wound healing assays and (C) the chamber assays were used to determine the effect of MSI2 on the migration and invasion of QBC939 cell. (B) Quantification of the wound healing assay was performed by measuring the covered areas of 48 hours compared with the uncovered areas of 0 hour. Magnification $200 \times$. (D) Quantification of migrated or invaded cells was performed by calculating the number of cells that pass through the membrane per field. $* * P<0.0 \mathrm{I}$, and $* * * P<0.00 \mathrm{I}$.

epithelial cell migration, as well as cell growth and survival. ${ }^{28}$ Guo et al concluded that MSI2 expression was markedly upregulated in both pancreatic ductal adenocarcinoma specimens and cell lines, and high MSI2 expression predicted poor outcomes in patients; thus, it can be used as a biomarker for accurate prognosis. ${ }^{30}$ To determine whether MSI2 is also a cancer-related gene in eCCA, we first measured its expression levels in human samples. As we predicted, both the mRNA and protein expression levels of MSI2 were increased in tumor tissues. Moreover, high MSI2 expression levels were an independent prognostic factor for decreased OS and DFS in patients with eCCA. These data suggested that MSI2 is a cancer-related gene participating in eCCA development and progression. In addition, as a prognostic biomarker of
eCCA, MSI2 expression data could be very useful for making clinical decisions.

eCCA is an invasive tumor with high mortality due to its late clinical presentation and lack of effective nonsurgical therapies. However, curative surgery is not suitable for most patients owing to the existence of distant dissemination when diagnosed. In the present study, we found that high MSI2 expression in patients was usually associated with lymph node metastasis and advanced TNM stage., ${ }^{4,31}$ Naturally, we predicted that MSI2 participated in eCCA migration and invasion. Due to its high expression in eCCA tumor cells, we knocked MSI2 down by lentivirus transfection. Interestingly, MSI2 knockdown not only inhibited growth but also repressed the migration and invasion ability of eCCA 
cells. Accumulating evidence has demonstrated that EMT, a process including gene expression modifications that allow concurrent epithelial phenotype repression and mesenchymal phenotype activation, is an early event in the distant metastasis of tumor cells. ${ }^{32,33}$ In addition, the hallmarks and regulators of EMT, such as E-cadherin, N-cadherin, SNAIL family proteins, TWIST $1 / 2$, and ZEB1/2, can be detected in human eCCA specimens, and their presence has been correlated with poor prognosis. ${ }^{26,34}$ To explore whether MSI2 promoted the invasion and metastasis of eCCA cells by inducing EMT,
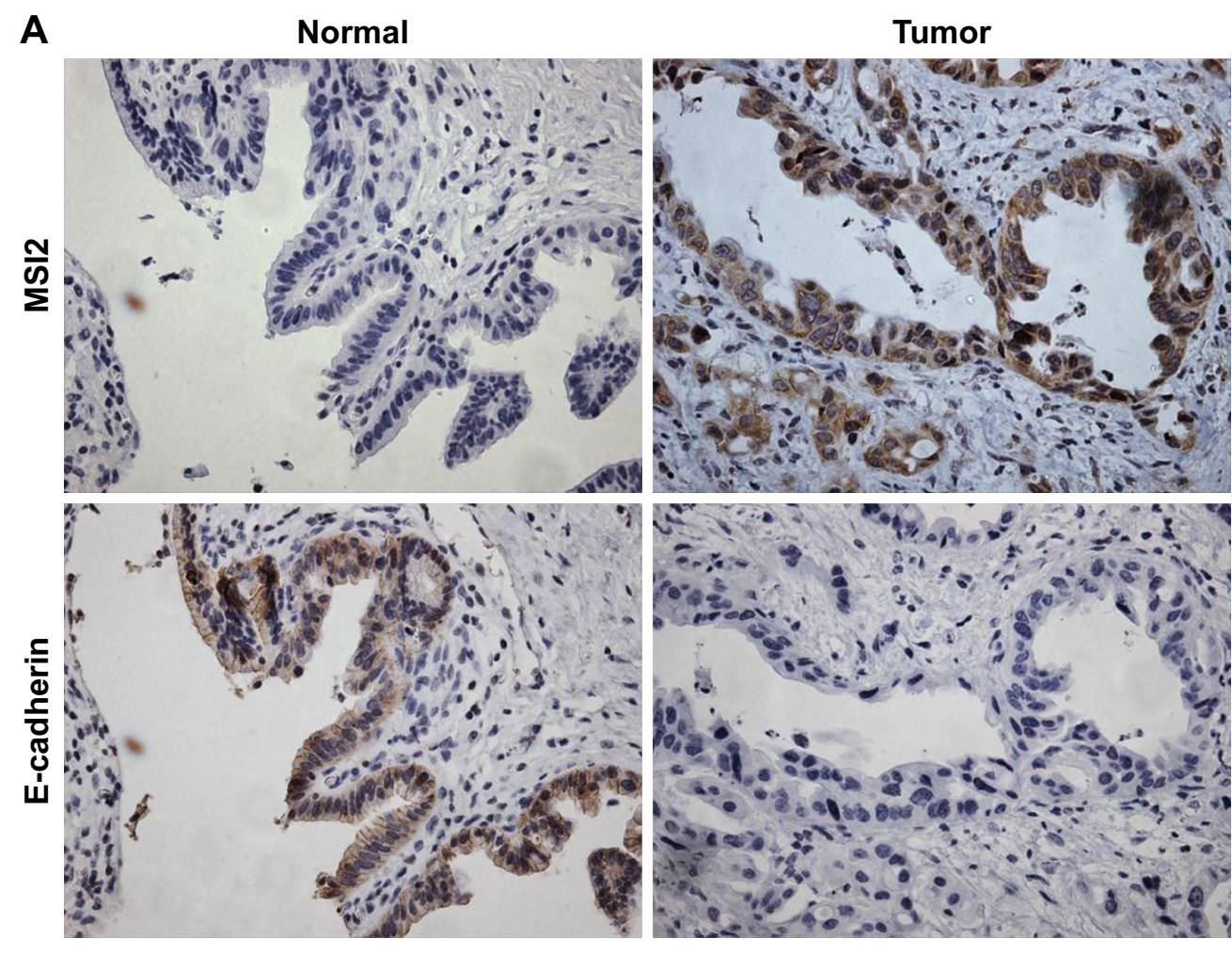

B

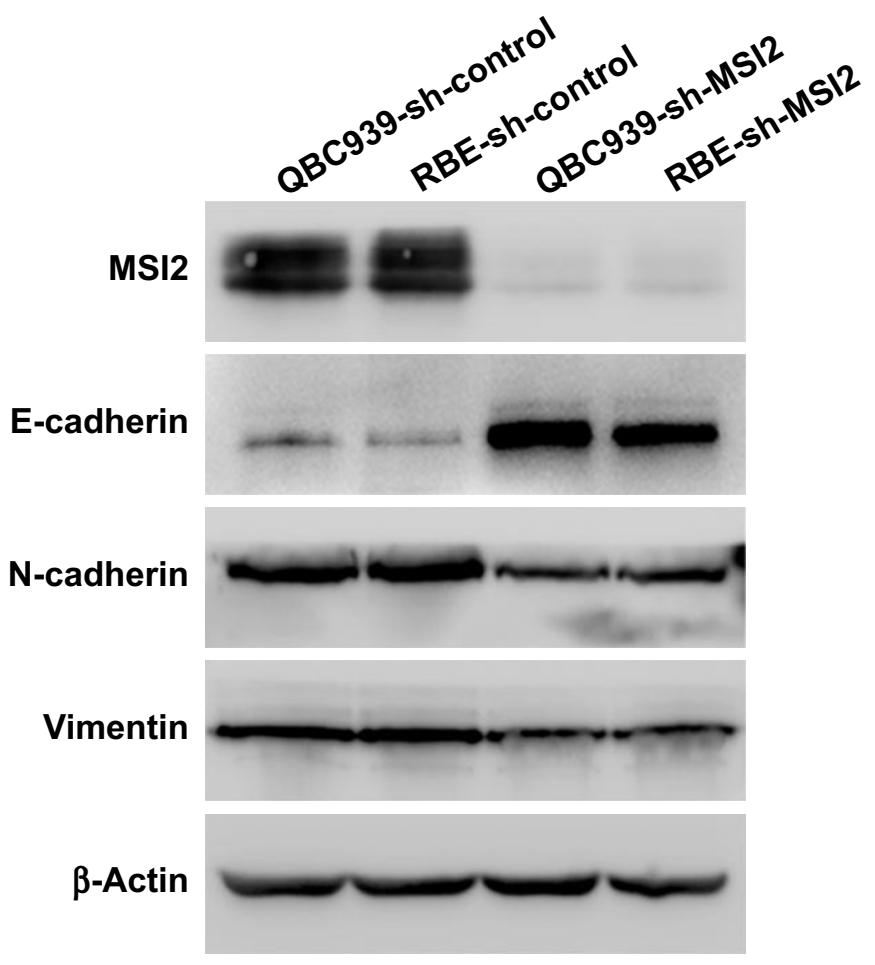

Figure 5 (Continued) 

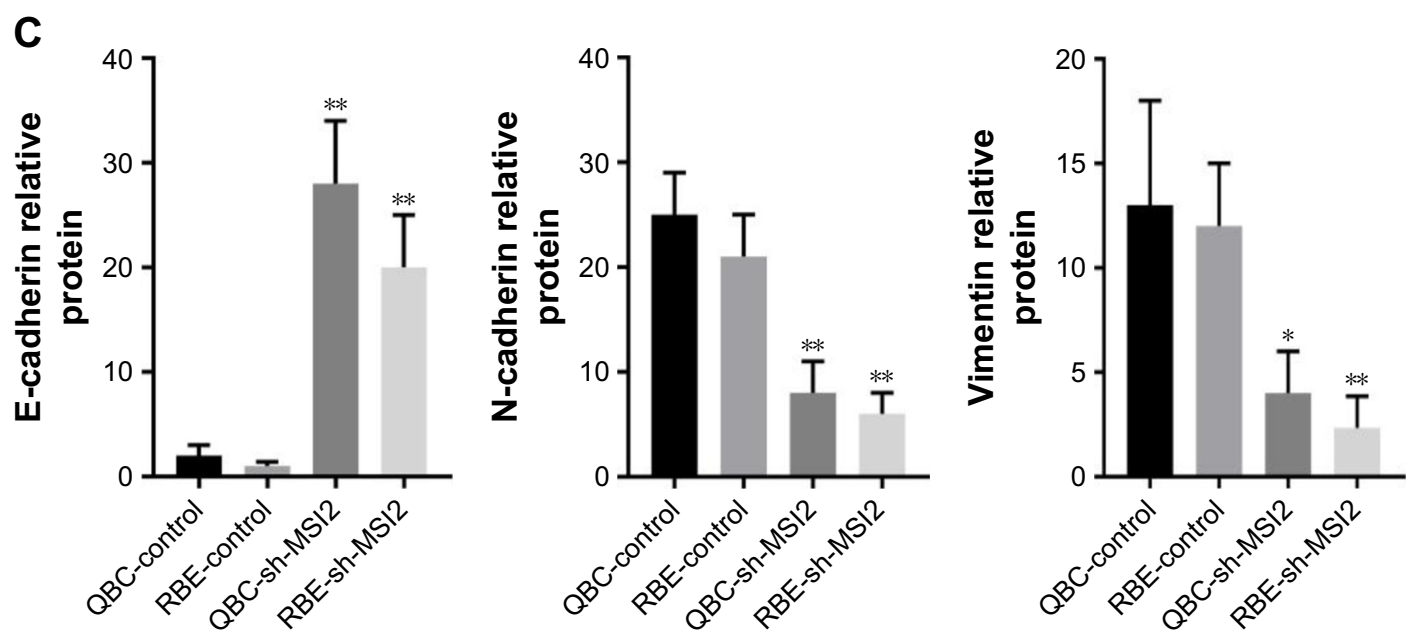

Figure 5 MSI2 induces EMT in extrahepatic cholangiocarcinoma.

Notes: (A) Representative IHC images of MSI2 and E-cadherin expression in eCCA tissues and normal bile duct. Magnification 200x. (B) Western blot analysis of expression of EMT-related markers in human eCCA cell lines with stably depleted MSI2. (C) Quantification of the blots with image software by calculating grayscale of the band. $* P<0.05$, and $* * P<0.01$.

Abbreviations: eCCA, extrahepatic cholangiocarcinoma; EMT, epithelial-mesenchymal transition; IHC, immunohistochemistry.

we detected EMT-related markers. Consistent with our prediction, MSI2 knockdown enhanced E-cadherin expression and decreased $\mathrm{N}$-cadherin and vimentin expressions, which meant that MSI2 promoted the translation of EMT-related proteins and enhanced EMT in eCCA.

Because of the high degree of malignancy and the lack of effective treatment for eCCA, finding tumor markers that could predict prognosis and guide clinical treatment is imperative. Our study identified that MSI2 could serve as a prognostic biomarker in eCCA patients and be used clinically in the future, although more prospective studies are needed to verify this proposal. In addition, the importance of MSI2 in malignant tumors has caused many researchers to attempt to develop small-molecule inhibitors of this protein..$^{21,35}$ Interestingly, our results also indicated that MSI2 was a potential therapeutic target for improving the prognosis of eCCA. At the same time, given the large amount of evidence that has emphasized the important role of EMT in eCCA development, EMT has also become an attractive therapeutic target for eCCA. Thus, our research also contributes to the exploration of drugs targeting EMT. There are some

Table 3 Correlations of MSI2 expression with E-cadherin in eCCA patients

\begin{tabular}{llcll}
\hline Immunoreactivity & \multicolumn{4}{c}{ MSI2 expression } \\
\cline { 2 - 5 } & Low & High & r-value & P-value \\
\hline E-cadherin expression & & & -0.586 & $<0.00$ I \\
Low & II & 38 & & \\
High & 24 & 5 & & \\
\hline
\end{tabular}

Abbreviation: eCCA, extrahepatic cholangiocarcinoma. limitations to our study. Though we propose that MSI2 could promote eCCA cell migration and invasion, we did not investigate the entire mechanism in our study. More experiments, both in vitro and in vivo, are needed to further determine the roles of MSI2 in the development and metastasis of eCCA.

Our study demonstrates, for the first time, that high MSI2 expression is an independent factor for poor prognosis in patients with eCCA. High MSI2 expression levels promote the growth and invasion of eCCA, whereas MSI2 downregulation represses the invasion and metastasis of eCCA through inhibiting EMT.

\section{Acknowledgments}

This study is supported by the National Natural Science Foundation of China (NO 81272397) and the Research Project of Anhui Natural Science for Colleges and Universities (KJ 2013Z143).

\section{Disclosure}

The authors report no conflicts of interest in this work.

\section{References}

1. Kennedy L, Hargrove L, Demieville J, et al. Recent advances in understanding cholangiocarcinoma. F1000Res. 2017;6:6.

2. Rizvi S, Gores GJ. Pathogenesis, diagnosis, and management of cholangiocarcinoma. Gastroenterology. 2013;145(6):1215-1229.

3. Razumilava N, Gores GJ. Cholangiocarcinoma. The Lancet. 2014; 383(9935):2168-2179.

4. Banales JM, Cardinale V, Carpino G, et al. Expert consensus document: cholangiocarcinoma: current knowledge and future perspectives consensus statement from the European Network for the Study of Cholangiocarcinoma (ENS-CCA). Nat Rev Gastroenterol Hepatol. 2016;13(5):261-280. 
5. Brivio S, Cadamuro M, Strazzabosco M, Fabris L. Tumor reactive stroma in cholangiocarcinoma: the fuel behind cancer aggressiveness. World J Hepatol. 2017;9(9):455-468.

6. Marin JJG, Lozano E, Herraez E, et al. Chemoresistance and chemosensitization in cholangiocarcinoma. Biochim Biophys Acta. 2018;1864 (4 Pt B):1444-1453.

7. Blechacz B, Gores GJ. Cholangiocarcinoma: advances in pathogenesis, diagnosis, and treatment. Hepatology. 2008;48(1):308-321.

8. Deoliveira ML, Cunningham SC, Cameron JL, et al. Cholangiocarcinoma: thirty-one-year experience with 564 patients at a single institution. Ann Surg. 2007;245(5):755-762.

9. Rosen CB, Heimbach JK, Gores GJ. Liver transplantation for cholangiocarcinoma. Transpl Int. 2010;23(7):692-697.

10. Sakakibara S, Nakamura Y, Satoh H, Okano H. Rna-binding protein Musashi2: developmentally regulated expression in neural precursor cells and subpopulations of neurons in mammalian CNS. J Neurosci. 2001;21(20):8091-8107.

11. Imai $\mathrm{T}$, Tokunaga $\mathrm{A}$, Yoshida $\mathrm{T}$, et al. The neural RNA-binding protein Musashi1 translationally regulates mammalian numb gene expression by interacting with its mRNA. Mol Cell Biol. 2001;21(12):3888-3900.

12. Kudinov AE, Karanicolas J, Golemis EA, Boumber Y. Musashi RNAbinding proteins as cancer drivers and novel therapeutic targets. Clin Cancer Res. 2017;23(9):2143-2153.

13. Kharas MG, Lengner CJ, Al-Shahrour F, et al. Musashi-2 regulates normal hematopoiesis and promotes aggressive myeloid leukemia. Nat Med. 2010;16(8):903-908.

14. Ito T, Kwon HY, Zimdahl B, et al. Regulation of myeloid leukaemia by the cell-fate determinant Musashi. Nature. 2010;466(7307):765-768.

15. Kwon HY, Bajaj J, Ito T, et al. Tetraspanin 3 is required for the development and propagation of acute myelogenous leukemia. Cell Stem Cell. 2015;17(2):152-164.

16. Griner LN, Reuther GW. Aggressive myeloid leukemia formation is directed by the Musashi 2/Numb pathway. Cancer Biol Ther. 2010; 10(10):979-982.

17. He L, Zhou X, Qu C, et al. Musashi2 predicts poor prognosis and invasion in hepatocellular carcinoma by driving epithelial-mesenchymal transition. J Cell Mol Med. 2014;18(1):49-58.

18. Kudinov AE, Deneka A, Nikonova AS, et al. Musashi-2 (MSI2) supports TGF- $\beta$ signaling and inhibits claudins to promote non-small cell lung cancer (NSCLC) metastasis. Proc Natl Acad Sci U S A. 2016; 113(25):6955-6960.

19. Lee J, An S, Choi YM, et al. Musashi-2 is a novel regulator of paclitaxel sensitivity in ovarian cancer cells. Int J Oncol. 2016;49(5): 1945-1952

20. Han Y, Ye A, Zhang Y, et al. Musashi-2 silencing exerts potent activity against acute myeloid leukemia and enhances chemosensitivity to daunorubicin. PLoS One. 2015;10(8):e0136484.
21. Minuesa G, Antczak C, Shum D, et al. A 1536-well fluorescence polarization assay to screen for modulators of the MUSASHI family of RNA-binding proteins. Comb Chem High Throughput Screen. 2014; 17(7):596-609.

22. Huang DW, Huang M, Lin XS, Huang Q. CD155 expression and its correlation with clinicopathologic characteristics, angiogenesis, and prognosis in human cholangiocarcinoma. Onco Targets Ther. 2017; 10:3817-3825.

23. Thiery JP, Acloque H, Huang RY, Nieto MA. Epithelial-mesenchymal transitions in development and disease. Cell. 2009;139(5):871-890.

24. Acloque H, Adams MS, Fishwick K, Bronner-Fraser M, Nieto MA. Epithelial-mesenchymal transitions: the importance of changing cell state in development and disease. J Clin Invest. 2009;119(6):1438-1449.

25. Vaquero J, Guedj N, Clapéron A, Nguyen Ho-Bouldoires TH, Paradis V, Fouassier L. Epithelial-mesenchymal transition in cholangiocarcinoma: from clinical evidence to regulatory networks. J Hepatol. 2017;66(2): 424-441.

26. Araki K, Shimura T, Suzuki H, et al. E/N-cadherin switch mediates cancer progression via TGF- $\beta$-induced epithelial-to-mesenchymal transition in extrahepatic cholangiocarcinoma. Br J Cancer. 2011;105(12): 1885-1893.

27. Ohyama T, Nagata T, Tsuda K, et al. Structure of Musashil in a complex with target RNA: the role of aromatic stacking interactions. Nucleic Acids Res. 2012;40(7):3218-3231.

28. Bennett CG, Riemondy K, Chapnick DA, et al. Genome-wide analysis of Musashi-2 targets reveals novel functions in governing epithelial cell migration. Nucleic Acids Res. 2016;44(8):3788-3800.

29. Fox RG, Park FD, Koechlein CS, Kritzik M, Reya T. Musashi signaling in stem cells and cancer. Annu Rev Cell Dev Biol. 2015;31:249-267.

30. Guo K, Cui J, Quan M, et al. The novel KLF4/MSI2 signaling pathway regulates growth and metastasis of pancreatic cancer. Clin Cancer Res. 2017;23(3):687-696.

31. Sirica AE, Gores GJ. Desmoplastic stroma and cholangiocarcinoma: clinical implications and therapeutic targeting. Hepatology. 2014; 59(6):2397-2402.

32. Baulida J. Epithelial-to-mesenchymal transition transcription factors in cancer-associated fibroblasts. Mol Oncol. 2017;11(7):847-859.

33. Puisieux A, Brabletz T, Caramel J. Oncogenic roles of EMT-inducing transcription factors. Nat Cell Biol. 2014;16(6):488-494.

34. Techasen A, Namwat N, Loilome W, et al. Tumor necrosis factor- $\alpha$ modulates epithelial mesenchymal transition mediators ZEB2 and S100A4 to promote cholangiocarcinoma progression. J Hepatobiliary Pancreat Sci. 2014;21(9):703-711.

35. Clingman CC, Deveau LM, Hay SA, et al. Allosteric inhibition of a stem cell RNA-binding protein by an intermediary metabolite. Elife. $2014 ; 3$. 


\section{Supplementary materials}

Table SI Differential expression of MSI2 in eCCA tissues and corresponding paracarcinoma tissues $(n=78)$

\begin{tabular}{llll}
\hline Tissue & MSI2 expression & P-value \\
\cline { 2 - 3 } & Low (\%) & High (\%) \\
\hline Carcinoma tissues & $35(44.9)$ & $43(55.1)$ & $21(26.9)$ \\
Paracarcinoma tissues & $57(73.1)$ & 0.001 \\
\hline
\end{tabular}

Abbreviation: eCCA, extrahepatic cholangiocarcinoma.

A

$$
\beta \text {-Actin }
$$$$
\mathbf{A}
$$$$
\text { sh-Contro }
$$
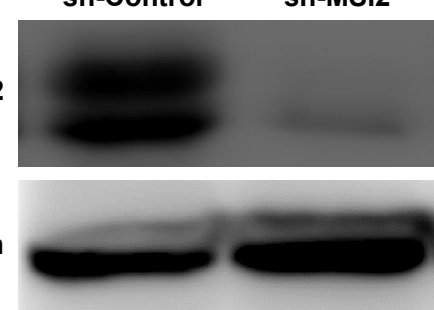

C

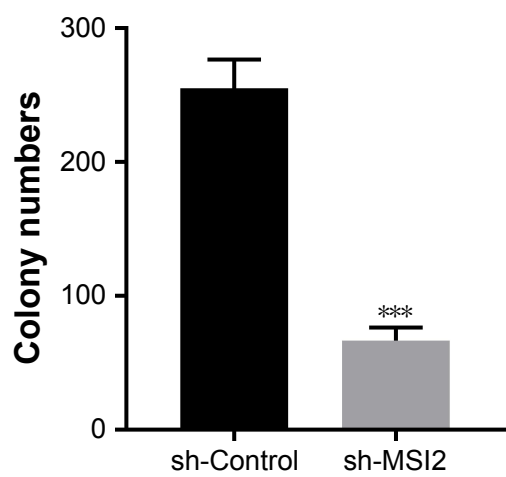

B
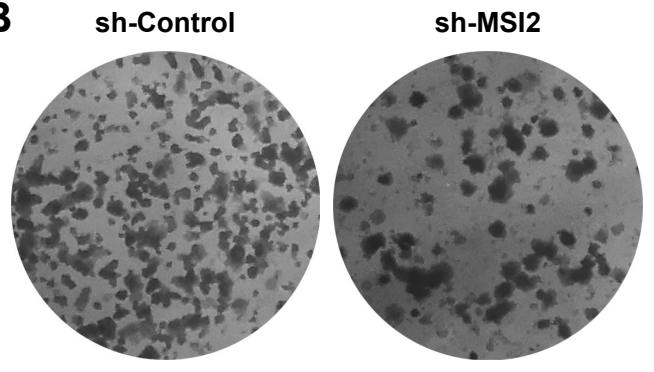

D

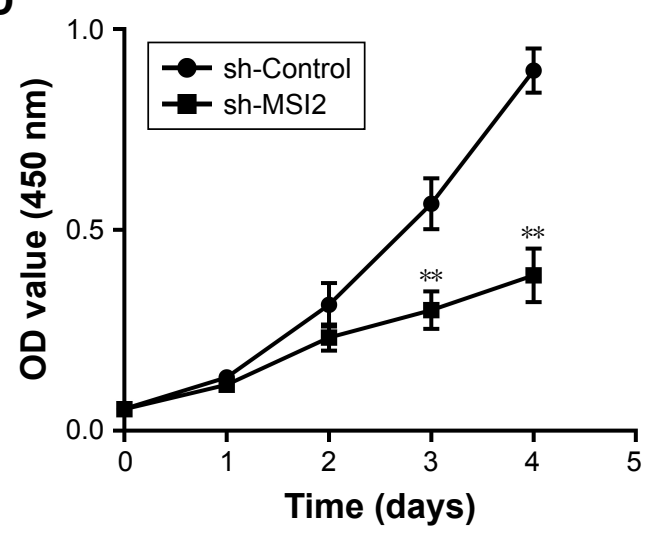

Figure SI MSI2 promotes extrahepatic cholangiocarcinoma cell growth.

Notes: (A) Verification of the efficiency of MSI2-shRNA plasmid in RBE cell line. (B) Colony formation assays show the effect of MSI2 on RBE cell growth and colony formation. (C) Quantification of colony formation assays through calculating colony numbers. (D) CCK-8 assay shows the effect of MSI2 on RBE cell proliferation. **P $<0.01$, and $* * * P<0.001$.

Abbreviation: CCK-8, Cell Counting Kit-8. 
A
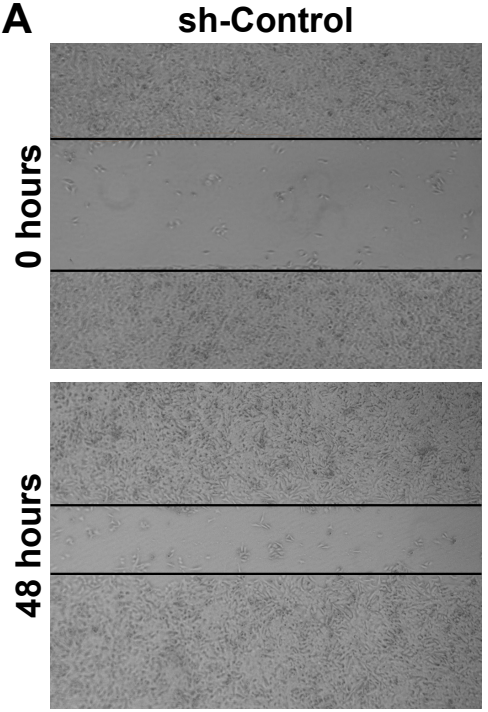

C

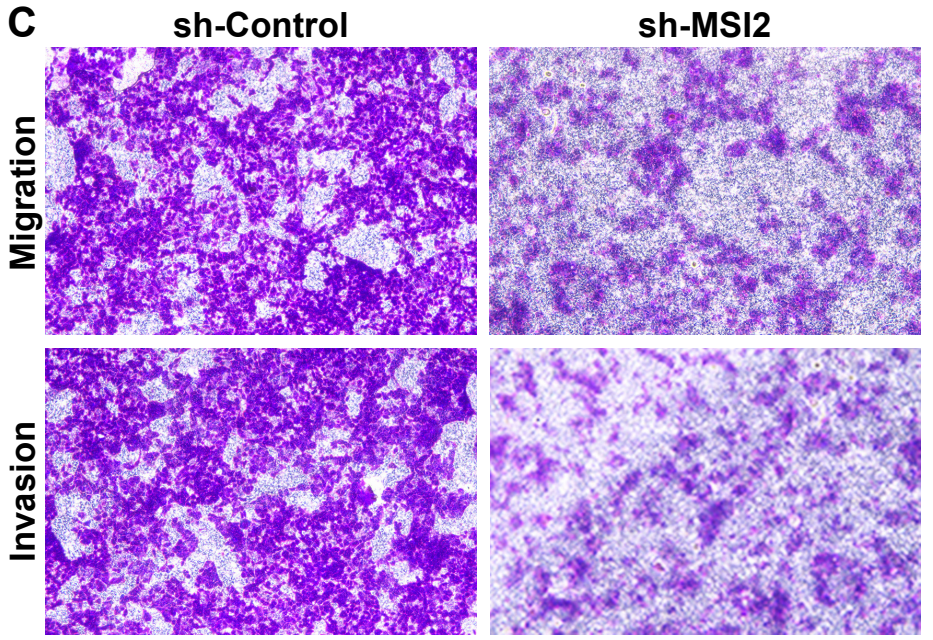

sh-MSI2
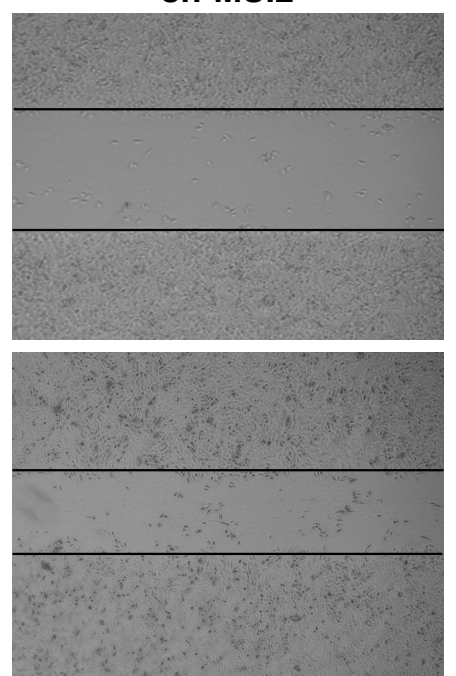

sh-MSI2

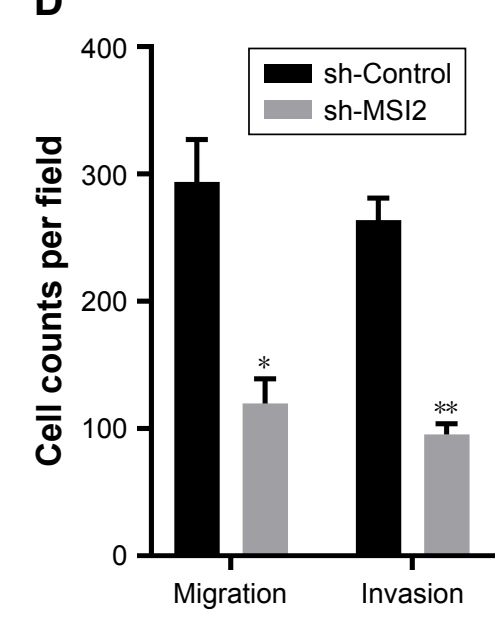

B

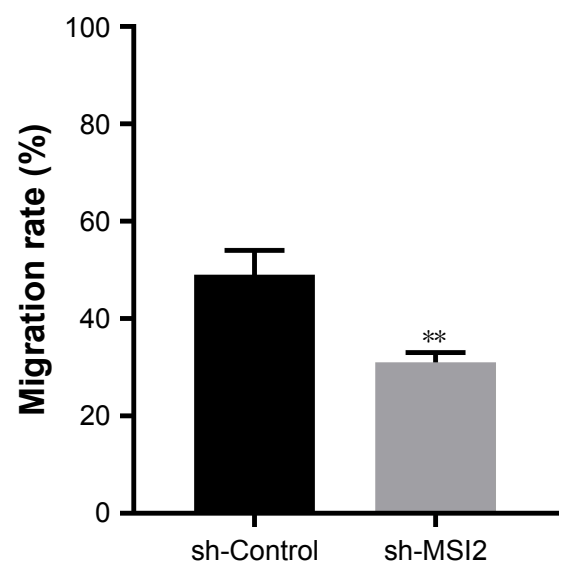

D

Figure S2 MSI2 promotes the migration and invasion of extrahepatic cholangiocarcinoma.

Notes: (A) Wound healing assays and (C) the chamber assays were used to determine the effect of MSI2 on RBE cells' migration and invasion. Magnification 200x. (B) Quantification of the wound healing assay was performed by measuring the covered areas for 48 hours compared with the uncovered areas of 0 hours. (D) Quantification of migrated or invaded cells was performed by calculating the number of cells that pass through the membrane per field. $* P<0.05$, and $* * P<0.01$.

\section{Publish your work in this journal}

OncoTargets and Therapy is an international, peer-reviewed, open access journal focusing on the pathological basis of all cancers, potential targets for therapy and treatment protocols employed to improve the management of cancer patients. The journal also focuses on the impact of management programs and new therapeutic agents and protocols on

Submit your manuscript here: http://www.dovepress.com/oncotargets-and-therapy-journal

patient perspectives such as quality of life, adherence and satisfaction. The manuscript management system is completely online and includes a very quick and fair peer-review system, which is all easy to use. Visit http://www.dovepress.com/testimonials.php to read real quotes from published authors. 